Results Pg infection increased PD-L1 expression on Het-1A cells within 24 hours of infection and increased PD-L1 mRNA within 4 hours of infection. PD-L1 expression level correlated with cellular bacterial burden on the cells in a dose-dependent manner. PD-L1 expression was decreased by the Kgp inhibitor, atuzaginstat, or an Rgp inhibitor, COR613, and PD-L1 expression was completely blocked when both gingipain inhibitors were used together (figure 1). Pg also induced expression of PD-L1 on the surface of infected SCC-25, SH-SY5Y, and RAW cell lines. Western blot analysis and qPCR revealed that Kgp inhibition, but not Rgp inhibition, was able to inhibit the non-canonical activation of b-catenin and down regulation of classical wnt pathway effectors at both the mRNA and protein level.
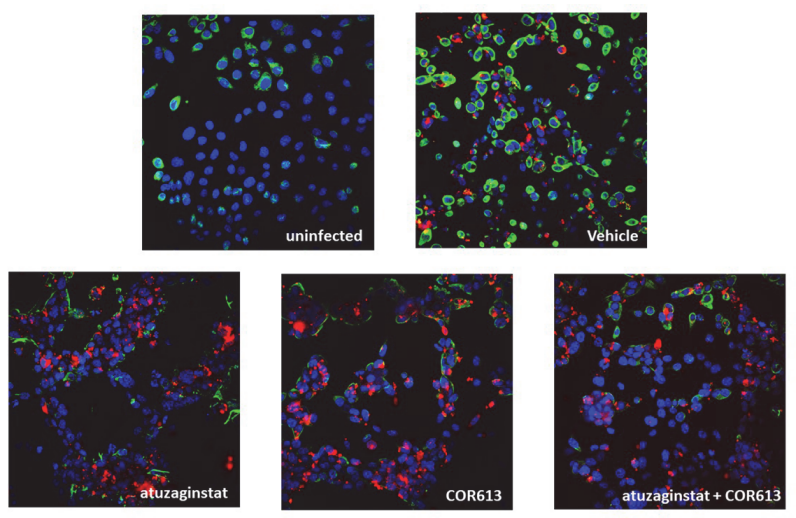

Abstract 676 Figure 1 Gingipain inhibitors block PD-L1 induced by $\mathrm{Pg}$

Pg grown with and labeled by red fluorescent membrane-incorporated dye was pre-treated with vehicle or the compounds listed for $30 \mathrm{~min}$. Het-1A cells were infected $(\mathrm{MOI}=20)$ for 24 hours, washed, fixed and stained for visualization of the nuclei (DAPI, blue), PD-L1 protein (antiPDL1 primary and secondary antibodies, green), and Pg infection (red). Images were captured with immunofluorescent confocal microscopy.

Conclusions In host cells infected with $\mathrm{Pg}$, gingipains mediate the induction of PD-L1 as a mechanism of immune evasion through the non-canonical activation of the wnt pathway. Further studies to elucidate induction mechanisms are in progress. In esophageal cancer and other cancers infected with Pg, combining gingipain inhibitors with anti-PD-1 therapy may improve treatment outcomes.

http://dx.doi.org/10.1136/jitc-2020-SITC2020.0676

\section{EVALUATION OF ANTI-PD1 EFFICACY IN GERM-FREE AND ANTIBIOTIC-TREATED SPF MICE BEARING MC38 TUMORS}

${ }^{1}$ Ying Jin, ${ }^{2}$ Xue Liang, ${ }^{3}$ Frieda Zhang, ${ }^{4}$ Guangmao Mu, ${ }^{4}$ Wei Zhou, ${ }^{4}$ Annie An, ${ }^{5}$ Marvin Ouyang, ${ }^{2}$ Cai Li, ${ }^{2}$ Andy Liaw, ${ }^{4}$ Henry Li, ${ }^{4}$ Davy Ouyang* ${ }^{1}$ Crown Bioscience Inc, San Diego, CA, USA; ${ }^{2}$ Merck Research Laboratories, Kenilworth, NJ, USA; ${ }^{3}$ Cyagen Biosciences Inc, Taicang, China; ${ }^{4}$ Crown Bioscience Inc., San Diego, CA, USA; ${ }^{5}$ Cyagen Biosciences Inc., Taicang, China

Background Increasing evidence has indicated the important role of gut microbes in mediating normal and pathologic immune responses to cancer in both patients and animal models. There is growing effort in modulating microbiota composition to improve the outcome of cancer immunotherapy. To investigate the immunomodulatory roles of microbiota-based therapeutics preclinically, germ-free (GF) mice are often used because they are free of microorganisms. However, logistic challenges and inherited physiological deficits in GF mice are also generally acknowledged. Alternative approach of depleting gut microbiota in using specific pathogen-free (SPF) mice with broad-spectrum antibiotics has also been adopted. Potential challenges with this approach are possible acquisition of antibiotic-resistant bacteria and potential expansion of fungi. Here we report on the efficacy assessment of anti-PD-1 mAb on MC38 syngeneic tumors in both GF mice and antibiotictreated SPF mice.

Methods C57BL/6 mice were inoculated subcutaneously with MC38 tumor cells. In the GF study, GF mice (Taconic, provided by Cyagen) were housed in germ-free isolators at a Cyagen facility, and a cohort of SPF mice (Taconic) were used as controls. Both GF and SPF mice were randomized for isotype or anti-PD-1 mAb (mDX400) treatment when the tumors were established $\left(80-120 \mathrm{~mm}^{3}\right)$ and were continuously monitored for tumor growth over time. In the antibiotic treatment study, different antibiotic regimens were administered to SPF mice (Lingchang) in drinking water starting 2 weeks prior to MC38 tumor inoculation and continued throughout the study. Mice were treated with vehicle control or anti-PD-1 mAb (RMP1-14; CrownVivo ${ }^{\mathrm{TM}}$ ).

Results Tumor growth is significantly faster in GF than SPF mice, and mDX400 slowed the tumor growth rate in both GF and SPF mice. The tumors achieved complete regressions on 4 out of $10 \mathrm{GF}$ mice as compared to 6 out of $10 \mathrm{SPF}$ mice, yet the difference of mDX400 efficacy in GF vs SPF mice did not reach statistical significance. In antibiotic-treated SPF mice, none of the antibiotic regimens showed significant impact on MC38 tumor growth nor anti-PD-1 efficacy in SPF mice, which was contrary to most reported data. Immune profiling on tumor infiltrating lymphocytes in these mice and microbiota analysis by $16 \mathrm{~S}$ rRNA gene amplicon sequencing are ongoing and the data will be presented at the meeting. Conclusions We have observed faster tumor growth in GF mice, however, the efficacy of anti-PD-1 antibody is not impacted by GF condition or treatment with broad-spectrum antibiotics. These results are different from previously published work, indicating high variability in preclinical models. Ongoing analysis with antibiotic-treated mice will provide further insight.

Ethics Approval Animal experiments were conducted in accordance with animal welfare law, approved by local authorities, and in accordance with the ethical guidelines of CrownBio (Taicang).

http://dx.doi.org/10.1136/jitc-2020-SITC2020.0677

\section{ADDITION OF A SINGLE BACTERIA FACILITATES ANTI- TUMOR IMMUNITY AND LONG-TERM SURVIVAL IN COLORECTAL CANCER}

${ }^{1}$ Abigail Overacre-Delgoffe*, ${ }^{1}$ Anthony Cillo, ${ }^{1}$ Hannah Bumgarner, ${ }^{1}$ Ansen Burr, ${ }^{2}$ Justin Tometich, ${ }^{2}$ Amrita Bhattacharjee, ${ }^{3}$ Tullia Bruno, ${ }^{3}$ Dario Vignali, ${ }^{1}$ Timothy Hand. 'University of Pittsburgh, Pittsburgh, USA; ${ }^{2}$ Children's Hospital of Pittsburgh, Pittsburgh, PA, USA; ${ }^{3}$ UPMC Cancer Center, Pittsburgh, PA, USA

Background Colorectal cancer remains one of the most common and deadliest cancers worldwide and effective therapies are lacking. While immunotherapy has revolutionized treatment for many cancers, the overwhelming majority of 
colorectal cancer patients are non-responsive and the 5-year survival rate for advanced disease is $<20 \%$. Immunotherapeutic response has been associated with select members of the microbiome in melanoma; however, the potential benefit in colorectal cancer and the underlying mechanisms remain unclear. We sought to determine how specific members of the intestinal microbiome affect anti-tumor immunity in colorectal cancer (CRC) in hopes of discovering novel treatments and revealing potential hurdles to current therapeutic response in CRC patients.

Methods We utilized a carcinogen-induced mouse model of CRC and colonized half of the tumor-bearing mice with Helicobacter hepaticus (Hhep) 7 weeks post AOM. Tumor number was assessed 12 weeks post AOM. We isolated lymphocytes from the lamina propria, colonic epithelium, mesenteric lymph nodes, and tumor(s) to track the spatial and transcriptional Hhep-specific and endogenous immune responses during tumor progression through 5' single cell RNAseq, flow cytometry, and immunofluorescence. In addition, we utilized $16 \mathrm{~S}$ sequencing and FISH to track Hhep colonization, location within the colon, and its impact on the surrounding microbiome.

Results We have found that rational modification of the microbiome of colon tumor-bearing mice through addition of a single bacteria, Hhep, led to tumor control or clearance and a significant survival advantage. Colonization led to the expansion of the lymphatic network and development of numerous peri- or intra-tumoral tertiary lymphoid structures (TLS) composed of Hhep-specific CD4 $\mathrm{T}$ follicular helper cells (TFH) as well as the bacteria itself. This led to an overall 'heating' of the tumor, wherein we saw an increase of CD4 T cell infiltration to the tumor core as well as an increase in CD103+ type 1 DC (cDC1) recruitment through increased chemokines such as CCL5 and XCL1. Hhep-specific TFH were both necessary and sufficient to drive TLS formation, increased immune invasion, and anti-tumor immunity.

Conclusions We have shown that addition of a single bacteria, Hhep, leads to a reduction in CRC tumor burden or clearance through lymphatic expansion, TLS formation, and remodeling of the tumor microenvironment, and that Hhepspecific $\mathrm{T}$ cells are required for tumor control. These studies suggest that rational modification of the microbiome and microbiome-specific $\mathrm{T}$ cells can positively impact anti-tumor immunity and may represent a unique immunotherapeutic target to turn resistant tumors into responsive tumors.

http://dx.doi.org/10.1136/jitc-2020-SITC2020.0678

\section{HIGH FIBER DIET MODIFIES GUT MICROBIOME, PROPIONATE PRODUCTION, INTRATUMOR IMMUNE RESPONSE AND IS ASSOCIATED WITH OUTCOME IN PATIENTS WITH LUNG CANCER TREATED WITH IMMUNE CHECKPOINT INHIBITORS}

Corentin Richard*, Myriam Benlaifaoui, Omar El Ouarzadi, Khoudia Diop, Antoine Desilets, Julie Malo, Wiam Belkaid, Andréanne Leblanc, Julien Lamontagne, Meriem Messaoudene, Arielle Elkrief, Bertrand Routy. CRCHUM, Montreal, Canada

Background The gut microbiome plays a key role in immune checkpoint inhibitors (ICI) efficacy and several strategies are currently being investigated to improve microbiome composition. The impact of a specific diet on microbiome modulation and clinical outcomes remains unknown. In this study, we assessed the effects of a high fiber diet on clinical outcomes as well as on microbiome composition, production of fecal metabolites, and intratumor immune infiltration in metastatic non-small cell lung cancer (mNSCLC) patients amenable to ICI.

Methods In this prospective study, 39 chemotherapy-refractory or naive patients with mNSCLC treated with ICI alone or in combination with chemotherapy completed a validated dietary survey. Based on the total fiber intake, patients were divided into high vs low fiber groups (HF vs LF). Objective response rate (ORR), progression-free survival (PFS) and overall survival (OS) were compared between both groups. In addition, fecal and tumor samples were collected prior to ICI initiation. Fecal metagenomic sequencing was performed and fecal short-chain fatty acids (SCFA) were measured by LC-MS/MS. Tumoral transcriptome profiling was performed through RNA sequencing to define differentially expressed pathways.

Results Baseline characteristics were well balanced between both groups, including body mass index (BMI) and PD-L1 status. Median PFS for the HF group was longer compared to the LF group (27.4 vs 12.6 months). Microbiome metagenomic profiling revealed higher baseline alpha diversity $(p=0.048)$ in the HF group compared to the LF group. Bifidobacterium, Alistipes, and Bacteroides salyersiae were enriched in the HF group while Fusobacterium was overrepresented in the LF group. SCFA measurement revealed that a high level of propionate correlated with a significantly longer OS (not reached vs 18.4. months, $\mathrm{p}=0.02$ ) in the entire cohort. Moreover, propionate levels were significantly higher in the HF vs LF group $(p=0.02)$. At the tumor level, RNA sequencing demonstrated a downregulation of DNA repair mechanisms and an upregulation of humoral and adaptive immune responses in the HF group.

Conclusions In this study, we demonstrated that a HF diet in patients with mNSCLC was associated with better clinical outcomes. Importantly, HF was associated with an enrichment of previously reported beneficial gut bacteria. Moreover, propionate correlated with longer OS and was increased in the HF group. This study provides further insights into how the diet can beneficially shift the microbiome composition and intratumor immune responses in patients with mNSCLC treated with ICI and this may lead to novel, dietary-geared therapeutic avenues in the oncomicrobiome arena.

Ethics Approval The study was approved by CRCHUM Institution,s Ethics Board, approval number 17.035.

http://dx.doi.org/10.1136/jitc-2020-SITC2020.0679

\section{AUTOMATED ION TORRENT BASED SOLUTION ENABLES ACCURATE GUT MICROBIOME QUANTIFICATION OF BACTERIAL SPECIES RELEVANT TO RESEARCH IN CANCER AND ITS RESPONSE TO IMMUNOTHERAPY}

Shrutii Sarda*, David Merrill, Heesun Shin, Anna McGeachy, Birgit Drews, Wing Lee, Rajesh Gottimukkala, Janice Au-Young, Fiona Hyland. Thermo Fisher Scientific, South San Francisco, USA

Background A low-cost targeted solution to profiling gut microbial diversity is sequencing of the 16S rRNA gene; however, it is often insufficient to gain species level resolution due to high homology across different bacteria. Therefore, we developed a first-of-its-kind targeted sequencing solution that supplements $16 \mathrm{~S}$ gene targets, with highly species-specific primers for a cohort of 73 bacteria associated with research in diabetes, cancer and its response to immunotherapy, 